\title{
Laparoscopic radical antegrade modular pancreatosplenectomy
}

\author{
Yoo-Seok Yoon, Ho-Seong Han, Jai Young Cho, YoungRok Choi, Jangkyu Choi \\ Department of Surgery, Seoul National University Bundang Hospital, Seoul National University College of Medicine, Seongnam-si, Gyeonggi-do \\ 463-707, Korea \\ Correspondence to: Ho-Seong Han, MD, PhD. Professor of the Department of Surgery, Seoul National University Bundang Hospital, 300 Gumi- \\ dong, Bundang-gu, Seongnam-si, Gyeonggi-do 463-707, Korea. Email: hanhs@snubh.org.
}

\begin{abstract}
Although laparoscopic distal pancreatectomy is widely accepted for benign or borderline malignant pancreatic diseases, its application for pancreatic ductal adenocarcinoma (PDAC) remains controversial. Several recent reports have shown that laparoscopic surgery, for the treatment of PDAC, is associated with similar postoperative complications and survival outcomes compared with open surgery, and offers several advantages, particularly shorter hospital stay and less blood loss. However, potential risk of bias cannot be excluded because these results were obtained in retrospective studies. More importantly, it is unclear whether the extent of surgical resection is comparable between laparoscopic and open distal pancreatectomy. The aim of this video article is to show the technical feasibility of laparoscopic surgery to reproduce open radical antegrade modular pancreatosplenectomy (RAMPS) in terms of the extent of surgical resection.
\end{abstract}

Keywords: Distal pancreatectomy; radical antegrade modular pancreatosplenectomy (RAMPS); laparoscopy; pancreatic ductal adenocarcinoma (PDAC)

Received: 22 April 2016; Accepted: 29 June 2016; Published: 22 July 2016.

doi: 10.21037/jovs.2016.07.07

View this article at: http://dx.doi.org/10.21037/jovs.2016.07.07

\section{Introduction}

Laparoscopic distal pancreatectomy is a widely accepted operative procedure for treating benign or borderline malignant diseases in the body or tail of the pancreas (1-3), but its role in treating pancreatic ductal adenocarcinoma (PDAC) is poorly defined. With ongoing improvements in laparoscopic techniques, several recent reports have shown that laparoscopic surgery may be safe and effective for the treatment of PDAC (4-8). Laparoscopic surgery was associated with less blood loss and shorter hospital stay compared with open surgery. In addition, laparoscopic surgery did not compromise the oncologic outcomes in terms of the margin negative rate, the number of harvested lymph nodes, or the survival rate. However, laparoscopic and open distal pancreatectomy were generally compared in retrospective studies with a high risk of bias and, importantly, the extent of surgical resection was not specified or was heterogeneous. Standardizing the extent of resection and the surgical technique is essential to avoid false results when the oncologic outcomes are compared for two procedures such as open and laparoscopic distal pancreatectomy. In this context, radical antegrade modular pancreatosplenectomy (RAMPS), which was introduced by Strasberg et al. (9) to improve the tumor-free retroperitoneal margin and achieve adequate lymph node dissection, is a reference procedure for comparing laparoscopic and open surgery. This is because the dissection plane and extent of lymph node dissection are clearly defined in RAMPS. This video article describes our laparoscopic posterior RAMPS procedure.

\section{Patient selection and workup}

The indication for laparoscopic RAMPS and the decision to perform anterior or posterior RAMPS were based on abdominal computed tomographic (CT) images obtained within 1 month before surgery. Laparoscopic RAMPS is performed if the tumor lacks evidence of invasion to other organs except the left adrenal gland, spleen, and splenic vessels. As suggested by Strasberg et al., anterior RAMPS is chosen when the tumor is confined within the pancreas without evidence of invasion to the posterior capsule of the 


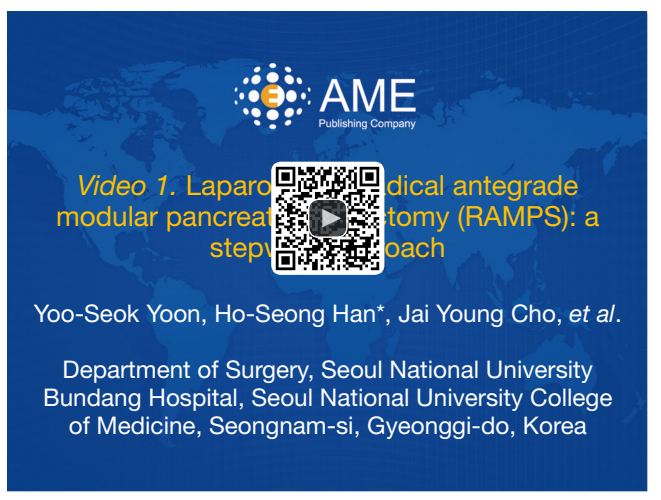

Figure 1 Laparoscopic radical antegrade modular pancreatosplenectomy (RAMPS): a stepwise approach (10). Available online: http://www.asvide.com/articles/1048

pancreas whereas posterior RAMPS is chosen when the tumor has penetrated the posterior capsule of the pancreas.

\section{Preoperative preparation}

Preoperative blood tests include a complete blood count, electrolytes, renal, liver function tests, and CA 19-9. Oral glucose tolerance test and glycosylated hemoglobin (HbA1c) are checked to detect undiagnosed diabetes mellitus associated with PDAC and to be used as a baseline in evaluating postoperative changes in glucose metabolism after pancreatectomy. Patients are allowed to consume food until midnight before the operation day. Mechanical bowel preparation is not performed. Antibiotic prophylaxis is administered within $30 \mathrm{~min}$ of incision. Written informed consent is obtained from all patients before surgery.

\section{Procedure}

Under general anesthesia, the patient is placed in a supine, $30^{\circ}$ reverse Trendelenburg position with leftside-up adjustment. After creation of a carbon dioxide pneumoperitoneum via a $12 \mathrm{~mm}$ infra-umbilical port, three or four additional trocars (12 and $5 \mathrm{~mm}$ trocars for the surgeon, and one or two $5 \mathrm{~mm}$ trocars for the assistant) were inserted. The two trocars for the surgeon are placed in the right upper abdomen, and trocars for the assistant are inserted in the left upper abdomen, as shown in the video (Figure 1). A three-dimensional flexible laparoscopic camera (Olympus, Tokyo, Japan) is used.

After trocar placement, the greater omentum is divided using LigaSure ${ }^{\circledR}$ (Medtronic, Minneapolis, MN, USA) from the midline towards the spleen. To maintain a good surgical field, the gastric antrum is fixed to the abdominal wall using a suture, which is pulled outside the abdominal wall. After elevating the neck of the pancreas using the grasper, the inferior pancreatic border is dissected until the superior mesenteric vein (SMV) is exposed. Small branches of the SMV encountered during dissection are controlled with LigaSure $^{\circledR}$. Thereafter, the surgeon approaches the superior border of the pancreas. The left gastric vein and artery are identified and followed toward the origin of the splenic artery. The left gastric lymph nodes are dissected until the splenic artery is exposed. The splenic artery is isolated and encircled with a vessel loop. A window is made between the pancreas and splenic vessels, and the neck of the pancreas is divided using an endoscopic stapler.

After transection of the pancreas, the splenic artery and vein are isolated and divided between Hem-o-lok clips $^{\circledR}$ (Weck Teleflex Medical, Research Triangle Park, NC, USA). Lymph nodes on the left side of the celiac axis and superior mesenteric artery (SMA) are dissected. The lateral and posterior sides of the SMA are dissected further until the left renal vein is identified. After further dissection along the left renal vein, the left adrenal vein is isolated and divided between metallic clips at the junction with the renal vein. Retroperitoneal dissection continues along the anterior surface of the renal vein laterally and posteriorly behind the adrenal gland. Posterior and lateral dissection is continued to the anterior surface of the kidney. The superior and inferior attachments of the pancreas are divided as dissection proceeds towards the spleen. The short gastric vessels are then divided. Finally, the lienorenal ligament is divided. A Jackson-Pratt drain is placed near the pancreatic stump. The surgical specimen is retrieved in a vinyl bag and extracted through a small incision created by extending a port-site incision.

\section{Postoperative management}

After surgery, the patients are transferred to a general ward without intensive care unit management unless they have underlying severe comorbidities requiring careful monitoring or they experience an intraoperative event. Early ambulation is encouraged from postoperative day (POD) 1 . Patients are allowed to drink water on POD 1, and have a soft blended diet from POD 2. Intravenous pain controllers are administered until POD 3-4 and oral analgesics are given thereafter if necessary. Abdominal CT is routinely performed on POD 5 to check for fluid collection around 
the pancreas stump or other intraabdominal complications. The abdominal drain is removed according to the drain amount, color, and amylase level, and the results of abdominal CT on POD 5. The patient is discharged if the following criteria are met: (I) abdominal drain has been removed; (II) the patient can tolerate a diet without needing intravenous fluid infusion; and (III) postoperative pain is tolerable with or without oral analgesics.

\section{Tips, tricks, and pitfalls}

- Fixation of the stomach to the abdominal wall can avoid the need for a trocar to maintain the surgical field;

- The gastrocolic ligament should be divided close to the gastroepiploic vessels so that bulky omentum on the side of the stomach does not block the surgical field;

- To decrease the risk of pancreatic fistula, the stapler cartridge should be selected according to the thickness and hardness of the pancreas. The stapling technique is also important. The closure jaw should be clamped slowly and carefully, taking more than $3 \mathrm{~min}$ at a fixed speed. If the pancreatic parenchyma is too hard to safely apply the stapler, the surgeon may transect the pancreas and ligate the exposed pancreatic duct instead;

- The course of the common hepatic artery and splenic artery should be carefully noted on the preoperative CT images. Sometimes, a tortuous hepatic artery can be mistaken for the splenic artery. The splenic artery should be divided at its origin from the celiac trunk after confirming the courses of the common hepatic artery and the left gastric artery;

- During lymph node dissection, the lymphatics or soft tissue around the lymph nodes should be grasped to avoid troublesome bleeding caused by lymph node injury;

- The left renal vein can be approached after lateral and posterior dissection of the left side of the SMA or superior dissection of the duodenojejunal junction. This vessel is the landmark of the inferior border of the dissection, and guides the proper plane of posterior dissection;

- The opening of the mesocolon, if present, should be repaired to avoid postoperative complications, particularly small bowl herniation through this opening.

\section{Acknowledgements}

None.

\section{Footnote}

Conflicts of Interest: The authors have no conflicts of interest to declare.

\section{References}

1. Venkat R, Edil BH, Schulick RD, et al. Laparoscopic distal pancreatectomy is associated with significantly less overall morbidity compared to the open technique: a systematic review and meta-analysis. Ann Surg 2012;255:1048-59.

2. Jin T, Altaf K, Xiong JJ, et al. A systematic review and meta-analysis of studies comparing laparoscopic and open distal pancreatectomy. HPB (Oxford) 2012;14:711-24.

3. Mehrabi A, Hafezi M, Arvin J, et al. A systematic review and meta-analysis of laparoscopic versus open distal pancreatectomy for benign and malignant lesions of the pancreas: it's time to randomize. Surgery 2015;157:45-55.

4. Kooby DA, Hawkins WG, Schmidt CM, et al. A multicenter analysis of distal pancreatectomy for adenocarcinoma: is laparoscopic resection appropriate? J Am Coll Surg 2010;210:779-85, 786-7.

5. Magge D, Gooding W, Choudry H, et al. Comparative effectiveness of minimally invasive and open distal pancreatectomy for ductal adenocarcinoma. JAMA Surg 2013;148:525-31.

6. Kang CM, Lee SH, Lee WJ. Minimally invasive radical pancreatectomy for left-sided pancreatic cancer: current status and future perspectives. World J Gastroenterol 2014;20:2343-51.

7. Croome KP, Farnell MB, Que FG, et al. Total laparoscopic pancreaticoduodenectomy for pancreatic ductal adenocarcinoma: oncologic advantages over open approaches? Ann Surg 2014;260:633-8; discussion 638-40.

8. Riviere D, Gurusamy KS, Kooby DA, et al. Laparoscopic versus open distal pancreatectomy for pancreatic cancer. Cochrane Database Syst Rev 2016;4:CD011391.

9. Strasberg SM, Drebin JA, Linehan D. Radical antegrade modular pancreatosplenectomy. Surgery 2003;133:521-7.

10. Yoon YS, Han HS, Cho JY, et al. Laparoscopic radical antegrade modular pancreatosplenectomy (RAMPS): a stepwise approach. Asvide 2016;3:286. Available online: http://www.asvide.com/articles/1048

doi: 10.21037/jovs.2016.07.07

Cite this article as: Yoon YS, Han HS, Cho JY, Choi Y, Choi J. Laparoscopic radical antegrade modular pancreatosplenectomy. J Vis Surg 2016;2:122. 